\title{
X-ray study of accretion flow in narrow-line Seyfert 1 galaxies
}

\author{
Yoshito Haba $^{1}$, Yuichi Terashima ${ }^{1}$ and Hideyo Kunieda ${ }^{1}$ \\ ${ }^{1}$ Institute of Space and Astronautical Science, 3-1-1 Yoshinodai, Sagamihara, 229-8510, Japan
}

\begin{abstract}
We analyzed 13 narrow-line Seyfert 1 galaxies (NLS1s) observed with XMM-Newton. Their X-ray continua are well parameterized by a combination of a hard power law and thermal emission from a standard Shakura-Sunyaev accretion disk. However, the observed disk temperatures are significantly higher than the prediction from the standard disk, and the temperatures are distributed within an extremely narrow range $(0.15-0.22 \mathrm{keV})$ in spite of the wide range of luminosities $\left(L_{\mathrm{X}}=10^{41-45} \mathrm{erg} \mathrm{s}^{-1}\right)$. Furthermore, we found an anti-correlation between the disk temperature and luminosity of PKS 0558-504. These results strongly indicate the breakdown of the standard accretion picture in NLS1s. We suggest that a slim disk, which is a stable disk solution under a high mass-accretion rate, could be able to explain the observational results, if the photon trapping effect is properly taken into account.
\end{abstract}

\section{Introduction}

Narrow-line Seyfert 1 galaxies (NLS1s) are key objects in order to understand a growth of a supermassive black hole, because it is thought that they harbor a relatively small $(\sim$ $10^{6-7} M_{\odot}$ ) black hole with a high mass-accretion rate (e.g., Pounds, Done, \& Osborne 1996; Mineshige et al. 2000). In the X-ray regime, they exhibit a prominent soft excess component, which is thought to be a hard tail of thermal emission from an accretion disk. Therefore, NLS1s can provide us with a unique opportunity to study an accretion flow under a very high mass-accretion rate. In this paper, we present results of spectral analyses of 13 NLS1s observed with XMM-Newton focusing on their soft excess component and discuss a possible origin of it in the framework of an accretion flow under a high mass-accretion rate regime.

\section{Results}

The hard $(>2 \mathrm{keV}) \mathrm{X}$-ray continua are well represented by a power law. If the hard continuum is extrapolated into the lower energy band, a soft excess component is found in all of the objects. In order to parameterize this soft component, we applied a multicolor disk blackbody (MCD) model (Mitsuda et al. 1984), which is a simple mathematical approximation of a standard Shakura-Sunyaev accretion disk (Shakura \& Sunyaev 1973). The data is adequately reproduced by this model, and we found that the observed temperatures are higher than the prediction from the standard disk model and are distributed within an extremely narrow range $(0.15-0.22 \mathrm{keV})$ in spite of the wide range of luminosities $\left(10^{41-45} \mathrm{erg} \mathrm{s}^{-1}\right)$. Furthermore, we found an anti-correlation between the temperature $k T$ and luminosity $L_{\mathrm{X}}$ of the most luminous NLS1 galaxy PKS 0558-504 (see Figure 1 left). These results strongly suggest that the standard accretion disk picture is no longer appropriate in the nucleus of NLS1s in our sample. 

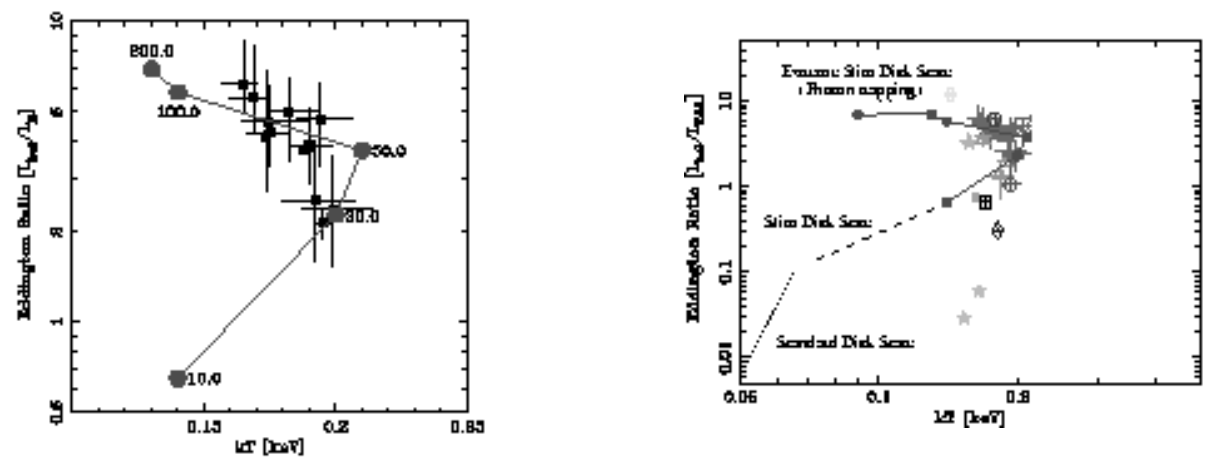

Figure 1. Left: Relationship between $k T$ and $\lambda_{\text {Edd }}$ of PKS 0558-504 (black). Gray line indicates the relation considering the photon trapping effect. Right: $k T-\lambda_{\mathrm{Edd}}$ distribution in our sample. Dotted and dashed lines imply a standard and a slim disk branch, respectively.

\section{Discussion and Conclusion}

It could be naturally explained why the observed temperatures are significantly higher than that expected from a standard disk model, if the accretion flow forms a slim disk (Abramowicz et al. 1988), which is expected when the mass-accretion rate $\dot{M}$ becomes much higher than that in the standard disk state. In the slim disk state, $k T-L_{\mathrm{X}}$ relation may be also different from the standard picture. Ohsuga et al. (2002) found that the radiative diffusion timescale becomes longer than the accretion timescale within the inner region of a super-critical accretion flow. Consequently, photons generated deeper inside the disk are trapped within the accretion flow and swallowed by a central black hole. This is so-called "photon trapping effect". Ohsuga et al. (2003) demonstrated that when the luminosity exceeds $\sim 2 L_{\mathrm{Edd}}$, the apparent disk temperature tends to decrease with increase of luminosity because of the enhanced photon-trapping effect. This can be recognized as follows: since higher energy photons tend to be generated deeper inside the accretion flow, they can be more effectively trapped than low energy photons. Thus, the high energy part of radiation is suppressed so that the emergent spectrum becomes softer. The filled circles in Figure 1 show the inferred $k T-\lambda_{\text {Edd }}\left(\equiv L_{\text {bol }} / L_{\text {Edd }}\right)$ relation considering the photon trapping effect. The numbers in the figure indicate the mass-accretion rate normalized by the critical mass-accretion rate $\dot{m} \equiv \dot{M} / \dot{M}_{\text {crit }}\left(\dot{M}_{\text {crit }} \equiv L_{\text {Edd }} / c^{2}\right)$. The observed behavior of $k T$ and $L_{\mathrm{X}}$ is well explained by the photon trapping effect. Right panel of Figure 1 displays $k T-\lambda_{\text {Edd }}$ distribution in our sample. Most objects may be in the extreme slim disk state, and the narrow distribution of $k T$ may be recognized by the fact that most objects just lie on the track where the disk temperature is saturated due to the photon trapping effect. The estimated mass-accretion rates of $10-100 \dot{M}_{\text {crit }}$ implies that the central mass can be $10^{8} M_{\odot}$ only within $10^{7-8}$ yrs. Therefore, NLS1s may be in a rapidly growing stage with a very high mass-accretion rate.

\section{References}

Abramowicz, M. A., et al. 1988, ApJ, 332, 646

Mineshige, S., et al. 2000, PASJ, 52, 499

Mitsuda, K., et al. 1984, PASJ, 36, 741

Ohsuga, K., et al. 2002, ApJ, 574, 315

Ohsuga, K., et al. 2003, ApJ, 596, 429

Pounds, K. A., et al. 1996, MNRAS, 277, 5

Shakura, N. I., \& Sunyaev, R. A. 1973, A\&A, 24, 337 